\title{
Ansiedad en matronas y enfermeras no especialistas de hospitales del servicio madrileño de salud
}

\author{
Diego Ayuso-Murillo ${ }^{a}$, Marina Jaime-Arranz $z^{b}$ \\ Ivan Herrera-Pecoc, Juan Gómez-Salgado d,e, \\ Juan Jesús García-Iglesias ${ }^{d}$, Carlos Ruiz-Frutos ${ }^{d, e}$
}

DOI: 10.12961/aprl.2019.22.04.02

Recibido: 8 de abril de 2019

Aceptado: 26 de julio de 2019

\section{RESUMEN}

Objetivo: El objetivo de este estudio es analizar los valores de la ansiedad estado y ansiedad rasgo en un grupo de enfermeras especialistas en ginecología y obstetricia (matronas) con respecto enfermeras asistenciales no especialistas.

Métodos: Se realizó un estudio descriptivo transversal, utilizando el cuestionario de ansiedad estado-rasgo (State-Trait Anxiety Inventory STAI), en un grupo de enfermeras no especialistas $(n=96)$ y matronas $(n=63)$ que desarrollan su actividad asistencial en hospitales del Servicio Madrileño de Salud en España.

Resultados: La ansiedad estado fue similar en las enfermeras no especialistas $(5,01 \pm 1,62)$ y en las matronas $(5,17 \pm 1,75)$. La ansiedad rasgo fue menor en las matronas $(3,46 \pm 1,58)$ que en el grupo de enfermeras no especialistas $(4,36 \pm 2,84)$, presentando éstas últimas mayores niveles de ansiedad habitual como rasgo de personalidad $(\mathrm{p}=0,013)$.

Conclusiones: Las matronas pueden presentar una mejor adaptación al estrés derivado de su trabajo que las enfermeras no especialistas, pudiendo estar relacionado con la posible mayor seguridad en el desarrollo de las funciones asistenciales en enfermería.

PALABRAS CLAVE: Especialidades de enfermería; Enfermera matrona; Ansiedad; Estrés laboral; STAI.

\section{ANXIETY IN NURSE MIDWIVES AND NON-SPECIALIST NURSES IN THE MADRID HEALTH SERVICE (SPAIN)}

\section{ABSTRACT}

Objective: We analyzed state anxiety and trait anxiety in a population of nurses specialising in gynecology and obstetrics (nurse midwives) and a group of generalist nurses.

Methods: Cross-sectional descriptive study using the State-Trait Anxiety Inventory questionnaire (STAI), administered to a group of non-specialist nurses $(n=96)$ and nurse midwives $(n=63)$ who practice in the Madrid Health Service of Spain.

Results: State anxiety was similar in generalist nurses and in midwives $(5,01 \pm 1,62$ and $5,17 \pm 1,75$, respectively). Levels of trait anxiety were lower in nurse midwives $(3,46 \pm 1,58)$ than in the non-specialist group $(4,36 \pm 2,84)$, with the latter presenting higher levels of habitual anxiety as a personality trait $(\mathrm{p}=0,013)$.

Conclusion: Nurse midwives may adapt better to the stress derived from their work than generalist nurses. This could be attributed to the greater training and safety that specialties provide for the development of nursing care functions.

KEYWORDS: Nursing specialties; nurse midwives; anxiety; occupational stress; STAI.

a. Consejo General de Enfermería de España, Madrid, España.

b. Hospital Universitario Puerta de Hierro Majadahonda, Madrid, España.

c. Facultad de Ciencias de la Salud en Universidad Alfonso X el Sabio,

Madrid, España.

d. Departamento de Sociología, Trabajo Social y Salud Pública,

Facultad de Ciencias del Trabajo, Universidad de Huelva, España.

e. Universidad Espíritu Santo, Guayaquil, Ecuador.
Correspondencia:

Prof. Dr. Juan Gómez-Salgado

Departamento de Sociología, Trabajo Social y Salud Pública.

Facultad de Ciencias del Trabajo. Universidad de Huelva.

Avda. Tres de Marzo, s/n, 21071 Huelva (España).

+0034699999168

salgado@uhu.es

Financiación:

Esta investigación no contó con financiación alguna, ni pública ni privada. Conflicto de intereses:

Los autores de esta investigación no tienen conflicto de interés, y lo planteado en la investigación es fruto del análisis de los resultados obtenidos. 


\section{INTRODUCCIÓN}

En España el desarrollo de las especialidades de enfermería, está enmarcado en el Real Decreto 450/2005', por el que el Sistema Nacional de Salud reconoce las especialidades de: Enfermería Obstétrico-Ginecológica (Matrona), Enfermería de Salud Mental, Enfermería Geriátrica, Enfermería del Trabajo, Enfermería de Cuidados Médico-Quirúrgicos, Enfermería Familiar y Comunitaria y Enfermería Pediátrica. La mayoría de las enfermeras españolas son en la actualidad diplomadas (3 años de formación universitaria) o graduadas (4 años de formación universitaria) y la diferencia entre una enfermera generalista (sin especialidad) y una enfermera especialista, es que está ultima completa su formación de forma específica con dos años más de formación como enfermera residente en su especialidad, con un contrato laboral de formación que incluye actividad teórica y práctica. Previo a este Real Decreto se promulgó el Real Decreto 992/1987², que contaba igualmente con siete especialidades de enfermería, pero que tuvo un escaso desarrollo ya que solo se pusieron en marcha las especialidades de Enfermería Obstétrico Ginecológica (Matrona) y Salud Mental, quedando el resto de especialidades sin desarrollar. Y previamente a este Real Decreto, hubo diferentes especialidades enfermeras, reguladas por diferente normativa, todas ellas derogadas con la entrada en vigor del citado RD 450/2005.

Las especialidades de enfermería en nuestro país después de catorce años del Real Decreto 450/2005', tienen un desarrollo escaso, con una implantación irregular en cuanto a las categorías profesionales dependiendo dicho desarrollo de las Comunidades Autónomas. Comunidades como Madrid, Cataluña, Euskadi o Castilla León solo tienen creada la categoría de Matrona dentro de las enfermeras especialistas y por una normativa europea de obligado cumplimiento, mientras que Baleares, Comunidad Valenciana, Castilla La Mancha, Canarias o Murcia son Autonomías ejemplo a seguir por el resto teniendo todas las especialidades creadas como categoría profesional. Siendo el siguiente paso necesario como normativa legal, el establecer el acceso a dichos puestos de trabajo la posesión del título de especialistas como requisito imprescindible e indispensable.

Las matronas son las enfermeras especialistas más representativas del colectivo profesional, ya que su especialidad es la más antigua e histórica de las actuales en la enfermería española ${ }^{3}$. La matrona está reconocida como un profesional que proporciona el apoyo necesario, los cuidados y los consejos durante el embarazo, el parto y el puerperio. Este cuidado incluye las medidas preventivas para evitar embarazos de riesgo, la promoción del parto eutócico, la detección de complicaciones en la madre y el niño, la planificación de cuidados, así como la ejecución de medidas de emergencia si fueran necesarias ${ }^{4}$.

La ansiedad es una de las sensaciones que con más frecuencia experimenta el ser humano. Tradicionalmente, ha sido definida como una emoción compleja, difusa y desagradable que se expresa por un sentimiento de temor y de tensión emocional, acompañada de un importante cortejo somático ${ }^{5}$.La ansiedad en el trabajo se asocia con el desempeño en entornos laborales, donde existe un escaso control y desarrollo competencial, o las situa-

Arch Prev Riesgos Labor 2019; 22 (4): $165-170$ ciones laborales son de excesiva presión asistencial, y pudiendo desencadenar el síndrome de burnout ${ }^{6-7-8}$.

El análisis del nivel de ansiedad en las enfermeras resulta de gran interés desde el punto de vista preventivo, ya que existen estudios que demuestran la correlación entre mayor ansiedad y aparición de depresión en enfermeras ${ }^{9}$ y se ha observado la existencia de una correlación entre estrés y síntomas depresivos ${ }^{10-12}$. Spielberger ${ }^{13-14}$ postula que una teoría adecuada de la ansiedad debe considerar este constructo como un proceso psicobiológico complejo, distinguiendo a su vez la ansiedad como un estado transitorio, o como un rasgo que permanece relativamente estable en determinadas personas. Este hecho derivó en la creación de uno de los cuestionarios existentes utilizados para medir la ansiedad, el "Cuestionario de ansiedad estado-rasgo" State-Trait Anxiety Inventory (STAI) ${ }^{14}$. En dicho cuestionario, la ansiedad estado (AE) es la que presenta el sujeto en el momento de rellenar el cuestionario. Por otro lado, la ansiedad rasgo (AR) hace referencia a las características de la personalidad relacionada con cuánto de ansioso está habitualmente el sujeto ${ }^{13}$. Esta sencillez a la hora de obtener mediciones claras de la ansiedad, unida a la obtención de diferentes evidencias de validez, hace del STAI uno de los cuestionarios más utilizados para evaluar la ansiedad en los entornos clínicos y laborales ${ }^{15-17}$.

En un estudio previo realizado por nuestro grupo con enfermeras clínicas y gestoras, se puso de manifiesto que las gestoras presentan una ansiedad estado algo menor pero muy similar a las clínicas. En el caso de la ansiedad rasgo se obtuvieron diferencias estadísticamente significativas, siendo menor en las gestoras, lo que podría estar relacionado con el perfil del puesto, el tipo de trabajo realizado y la necesidad de mayor tolerancia al estrés ${ }^{18}$.En este sentido, no se han encontrado estudios que analicen la ansiedad en enfermeras, relacionado con las variables de ser especialista o no, y se considera que podría ser un tema interesante a tener en cuenta, sobre todo desde el punto de vista de la actuación en gestión para la prevención de su aparición, planteando estrategias de monitorización y de actuación si fuera necesario ${ }^{19}$. En contraste con lo anterior, una enfermera generalista puede desarrollar su actividad en diferentes unidades asistenciales, con una variedad mucho mayor de pacientes a los que atender y cuidados que realizar, estando también sujetos a una movilidad mayor dentro de los hospitales, con la consiguiente necesidad de tener que adaptarse a diferentes puestos de trabajo y contextos laborales que las enfermeras especialistas, pudiendo ocasionar un mayor estrés laboral y niveles de ansiedad diferentes a las enfermeras especialistas ${ }^{20-21}$.

Por tanto, el objetivo de este estudio fue analizar los valores de la ansiedad estado (AE) y ansiedad rasgo (AR) en enfermeras especialistas en ginecología y obstetricia (matronas), con respecto a otro compuesto por enfermeras asistenciales generalistas.

\section{MÉTODOS}

Se realizó un estudio descriptivo transversal llevado a cabo en el periodo comprendido desde agosto a noviembre del año 2018. La población de estudio se constituyó por enfermeras no especialistas y matronas, que desempeñan su actividad en hospitales de la sanidad pública del Servicio Madrileño de Salud (SERMAS). Todas las participantes desarrollan su actividad a nivel asistencial. 
Los criterios de inclusión en el estudio fueron: participación voluntaria, tener el título de diplomada o graduada en Enfermería y/o ser especialista en obstetricia y ginecología (matrona), y encontrarse en activo. Las enfermeras se dividen en dos grupos de estudios; i) enfermeras especialistas matronas (EEM) y ii) enfermeras sin especialidad (ESE). En la Tabla 1, se especifican los veinte hospitales públicos del SERMAS que cuentan con maternidad (tienen enfermeras no especialistas y matronas) y que participaron en este estudio.

\section{TABLA 1}

Listado de Hospitales del Servicio Madrileño de Salud

de la Comunidad de Madrid participantes en el estudio.

Hospital Universitario Puerta de Hierro

Hospital Clínico

Hospital de Alcorcón

Hospital Infanta Cristina

Hospital de La Paz

Hospital Infanta Sofía

Hospital Gregorio Marañón

Hospital del Tajo

Hospital Infanta Leonor

Hospital Santa Cristina

Hospital de Getafe

Hospital Severo Ochoa

Hospital de Fuenlabrada

Hospital Ramón y Cajal

Hospital 12 de Octubre

Hospital de El Escorial

Hospital del Henares

Hospital de Arganda

Hospital de Móstoles

Hospital Príncipe de Asturias

En cuanto a la estimación del tamaño muestral, se partió de un universo compuesto por 15.110 ESE y de 695 EEM $^{22}$, en los hospitales públicos detallados en la tabla 1. A la hora de realizar el cálculo del tamaño muestral, se definió un nivel de confianza del 95\% (valor de 1,96), asimismo se considera que nos encontraremos ante la situación más desfavorable posible en cuanto a la proporción esperada del fenómeno a estudio $(\mathrm{p}=\mathrm{q}=50 \%)$. El porcentaje de error muestral aceptado en el presente trabajo fue del $12 \%$. En el presente trabajo de investigación se encuentra que, a la hora de estimar el tamaño de la población, se requerían no menos de 66 participantes para ESE y 61 participantes para EEM. Se obtuvo, finalmente, una muestra de 96 participantes para EES y de 63 participantes para EEM, asegurando así la representatividad de las muestras. El método de muestreo utilizado fue el probabilístico por conglomerados, considerando a cada uno de los hospitales públicos antes comentado, como un conglomerado. Dentro de cada conglomerado u hospital, se identifican dos estratos bien diferenciados que se han definido como: i) enfermeras asistenciales no especialistas y ii) enfermeras especialistas matronas.

Todas las matronas realizan su actividad en el servicio de paritorio y urgencias obstétricas; por el contrario, las enfermeras no especialistas pertenecían a los diferentes servicios asistenciales donde no hay especialidad de enfermería en la actualidad, como es el caso de hospitalización, Urgencias, Unidad de Cuidados Intensivos (UCI) y área ambulatoria.

Una vez definida esta información, se procedió a enviar toda la documentación relativa al proyecto de investigación, que incluye la hoja de Consentimiento Informado del voluntario y el cuestionario STAI, a las direcciones de Enfermería de cada Hospital. A la hora de solicitar que pudieran hacer llegar esta solicitud a los profesionales de enfermería que desempeñan sus funciones en cada hospital, se solicita a las Direcciones de enfermería, que las selecciones de los profesionales las realicen al azar, de modo que cualquier profesional, de cada uno de los dos grupos ya definidos anteriormente, tenga las mismas probabilidades de ser seleccionados.

Se procedió a valorar la AE y la AR mediante la cumplimentación del cuestionario STAI. El cuestionario STAI consta de 40 preguntas en una escala tipo Likert con 4 alternativas de respuesta cada una. Las 20 primeras preguntas del cuestionario constituyen la escala AE con la que el sujeto puede escribir como se siente en un momento particular, mientras que las siguientes 20 preguntas son la escala AR, en las que se pregunta cómo se siente el sujeto generalmente.

El STAI fue validado como instrumento fiable para medir la ansiedad en el contexto español por Seisdedos ${ }^{23}$, siendo la puntuación media tanto para la AE como para la AR en el STAI para la población española, es de 5,5 puntos (por encima de 5,5 puntos se considera superior a la media poblacional y por debajo de 5,5 puntos, menor a la media poblacional) ${ }^{23-24}$.

Las variables recogidas se pueden clasificar en: a) sociodemográficas: edad y sexo (variables moderadoras); b) laborales: nivel de especialidad de los participantes (variable dependiente) y años de experiencia en el puesto de trabajo (variable moderadora); c) factores relacionados con la ansiedad: AR y AE (variable independiente).

En cuanto al tratamiento y análisis de los datos, se utilizó el paquete de análisis estadístico SPSS v23 (IBM, USA). Se calculó estadística descriptiva para frecuencias y se determinó Chi cuadrado o t de Student para comparación entre grupos (EEM y ESE). Si fallan los test de normalidad se usará el test de MannWhitney. En el caso de que alguna de las variables no se distribuyese de forma normal, se procedió a realizar un enfoque de dos pasos mediante su transformación y normalización, utilizando el método descrito por Templeton en $2011^{25}$. Utilizando análisis univariado de la varianza para valorar la posible influencia de las variables. El nivel de significación se estableció en $\mathrm{p}<0,05$.

Todas las enfermeras que decidieron voluntariamente participar en el estudio, se les presentó una hoja informativa del proyecto para que conocieran de forma exhaustiva en qué consistía y su participación y se les solicitó el consentimiento informado. El proyecto de investigación fue evaluado y aprobado por el Comité Ético de Investigación Clínica del Hospital Universitario de Getafe, con la referencia $\mathrm{A}^{11-15}$. 


\section{RESULTADOS}

Datos sociodemográficos

En el presente estudio participaron un total de 159 participantes donde se contabilizaron un total de 63 (39,62\% del total) enfermeras matronas (EEM) y 96 (60,38\% del total) enfermeras con actividad asistencial que no refieren especialidad (ESE).Se procedió a comparar las características recogidas en el cuestionario: edad, sexo y tiempo de experiencia en el puesto actual (medido en años), con el objeto de observar si existía alguna diferencia inicial entre los grupos que pudiera influenciar en la AR y AE (tabla 2). En este sentido no se encontraron diferencias estadísticamente significativas al comparar ambos grupos (ESE y EEM), donde edad $(p=0,187)$, sexo $(2=0,422)$ y el tiempo de trabajo en la posición actual $(\mathrm{p}=0,536)$.

\section{TABLA 2}

Características generales sociodemográficas de la población de estudio.

\begin{tabular}{llllll} 
& \multicolumn{2}{l}{$\begin{array}{l}\text { Enfermeras no } \\
\text { especialistas }\end{array}$} & \multicolumn{3}{l}{ Matronas } \\
\hline Variables & $\mathbf{n}$ & $(\%)$ & $\mathbf{n}$ & $(\%)$ & $\mathbf{p}$ \\
\hline Sexo & 77 & $(86,1)$ & 58 & $(92,1)$ & 0,422 \\
\hline Mujer & 19 & $(13,9)$ & 5 & $(7,9)$ & \\
\hline Hombre & $(8,6)$ & 41,4 & $(10,1)$ & 0,187 \\
\hline $\begin{array}{l}\text { Edad (media DE) } \\
\text { 39,4 }\end{array}$ & $(2,11)$ & 7 & $(2,3 ; 12,5)$ & $0,536^{a}$ \\
$\begin{array}{l}\text { Experiencia en } \\
\text { puesto actual } \\
\text { mediana P25; P75) }\end{array}$ & 7 & & & & \\
\hline
\end{tabular}

DE: Desviación estándar. P25: Percentil 25. P75: Percentil 75. a. U de Mann-Withney.

\section{Análisis de los niveles de ansiedad}

En el análisis de datos, se observa que en el caso de enfermeras no especialistas aparecía una $A E$ de $5,01 \pm 1,62$ y una $A R$ de $4,36 \pm 2,84$, mientras que para las matronas la $\mathrm{AE}$ fue de $5,17 \pm 1,75$ y la AR de $3,46 \pm 1,58$. No se observan diferencias estadísticamente significativas entre la AE de ambos grupos $(p=0,447)$, sin embargo, sí aparece al comparar la AR de ambos grupos $(p=0,013)$ (tabla 3). Con el objetivo de valorar el posible efecto de las variables incluidas en el análisis, sexo, edad y años de experiencia con respecto a $A E$ y $A R$, en función de los grupos analizados ESE y EME, se observa que en función de la $\mathrm{AE}$ no existen diferencias en función de la especialidad $(\mathrm{P}=0,814)$ tras incluir en el análisis las covariables de edad, años de experiencia y sexo.

Por otro lado, al analizar la AR se observan diferencias estadísticamente significativas entre los grupos de especialidad tras analizar la posible influencia del sexo, edad y años de experiencia $(\mathrm{P}=0.017)$. La principal influencia es atribuida al sexo $(\mathrm{P}=0.019)$ en el modelo ajustado. Se analizó el sexo, en un análisis donde la variable dependiente fue al ansiedad (AR o AE) en función de la especialidad y controlando los años de experiencia y la edad. En esta situación se encontró que para el caso de la AR el sexo masculino era significativo $(p=0,033)$ mientras que no lo era para el sexo femenino $(p=0,062)$ (tabla 4$)$.

\section{DISCUSIONES}

De los resultados obtenidos, destaca que la $\mathrm{AE}$ es similar en ambos grupos de estudio, es decir en el momento de rellenar el cuestionario, tanto las enfermeras no especialistas como las matronas presentan un nivel de ansiedad similar, mientras que la AR en las matronas es menor que en el grupo de enfermeras no especia-

TABLA 3

Comparación de los niveles de ansiedad estado y ansiedad riesgo en los grupos de matronas y enfermeras no especialistas de hospitales.

\begin{tabular}{llllllll} 
Variables & Grupos & Media & $(\mathrm{DE})$ & Mediana & $(\mathrm{P} 25 ;$ & P75) & P \\
\hline Ansiedad estado & Enfermeras no especialistas & 5,01 & $(1,62)$ & 5 & $(4 ;$ & $3)$ & $0,447^{\mathrm{a}}$ \\
& Matronas & 5,17 & $(1,75)$ & 5 & $(4 ;$ & $6)$ \\
\hline Ansiedad riesgo & Enfermeras no especialistas & 4,36 & $(2,84)$ & 4 & $(3 ;$ & $5)$ & $0,013^{\mathrm{a}}$ \\
& Matronas & 3,46 & $(1,58)$ & 3 & $(2 ;$ & $5)$ \\
\hline
\end{tabular}

DE: Desviación estándar. P25: Percentil 25. P75: Percentil 75. a. U de Mann-Withney.

\section{TABLA 4}

Comparación de los niveles de ansiedad estado y ansiedad riesgo en los grupos de matronas y enfermeras no especialistas de hospitales por sexo.

\begin{tabular}{|c|c|c|c|c|c|c|c|}
\hline Variables & Sexo & Grupos Media & (DE) & Mediana & (P25; & P75) & $\mathrm{P}$ \\
\hline Ansiedad estado & $\begin{array}{l}\text { Hombre } \\
\text { Mujer }\end{array}$ & $\begin{array}{l}\text { Enfermeras no especialistas } \\
\text { Matronas } \\
\text { Enfermeras no especialistas } \\
\text { Matronas }\end{array}$ & $\begin{array}{l}13,17(1,73) \\
15,47(3,8) \\
14,78(0,96) \\
14,84(1,07)\end{array}$ & $\begin{array}{l}10 \\
14,5 \\
12 \\
14\end{array}$ & $\begin{array}{l}(8 ; \\
(11,5 \\
(8 ; \\
(10\end{array}$ & $\begin{array}{l}24,5) \\
19) \\
18) \\
17,5)\end{array}$ & $\begin{array}{l}0,59^{\mathrm{a}} \\
0,969^{\mathrm{a}}\end{array}$ \\
\hline Ansiedad riesgo & $\begin{array}{l}\text { Hombre } \\
\text { Mujer }\end{array}$ & $\begin{array}{l}\text { Enfermeras no especialistas } \\
\text { Matronas } \\
\text { Enfermeras no especialistas } \\
\text { Matronas }\end{array}$ & $\begin{array}{l}13,78(1,51) \\
7,45(3,32) \\
16,28(0,88) \\
13,817(0,96)\end{array}$ & $\begin{array}{l}12 \\
7 \\
13 \\
12\end{array}$ & $\begin{array}{l}(8,5 \\
(2,8 \\
(9 ; \\
(8 ;\end{array}$ & $\begin{array}{l}16) \\
9,8) \\
17) \\
18)\end{array}$ & $\begin{array}{l}0,033^{\mathrm{a}} \\
0,062^{\mathrm{a}}\end{array}$ \\
\hline
\end{tabular}

DE: Desviación estándar. P25: Percentil 25. P75: Percentil 75. a. U de Mann-Withney. 
listas, pudiendo estar relacionado con el puesto de trabajo desarrollado como especialista enfermera en el ámbito de la obstetricia y la ginecología. No se han encontrado estudios similares al nuestro en el cual se compare el nivel de ansiedad ligado a la variable de ser enfermera especialista o no especialista por tanto no se puede comparar en este sentido con otros estudios de investigación.

Tanto la AE como la AR están por debajo de la media de la población española, que está situada en 5,5 puntos para adultos tanto hombres como mujeres18, por lo que no parece que la ansiedad sea un factor de riesgo en las enfermeras del presente estudio, siendo dichos resultados diferentes a los encontrados en otros estudios como los de Ribeiro y Flores ${ }^{11-12}$. Pero se consideran de gran interés monitorizar y medir la ansiedad en las enfermeras de forma sistematizada, ya que como demuestran otros autores la ansiedad se asocia a estrés y esto genera síndrome de burnout, siendo un problema de salud laboral relevante ${ }^{6-7}$.

Los entornos positivos para la práctica profesional en enfermería se caracterizan por: políticas de salud, seguridad y bienestar en el trabajo, unas cargas de trabajo y unas demandas/tensiones del puesto que son equitativas y soportables, identidad profesional, autónoma y control de la práctica, seguridad del puesto de trabajo o niveles seguros de dotación personal entre otros ${ }^{25}$.

Se plantea como hipótesis que una enfermera con una especialidad como las matronas, tiene garantizado el desarrollar una actividad asistencial con mayor formación específica, con mayores competencias $^{26}$ y esto se puede traducir en trabajar en un entorno laboral más seguro, con menor nivel de estrés y por tanto que genere menor ansiedad a los profesionales que desarrollan esa actividad asistencial. Las enfermeras no especialistas no tienen las mismas condiciones laborales en la mayoría de los factores relacionados con los entornos positivos, ya que la seguridad en el puesto de trabajo, la identidad profesional con el puesto o las demandas/tensiones del puesto suelen ser diferentes a las matronas, que tienen mayores niveles de seguridad en su puesto de trabajo, una mayor identidad profesional o un nivel más seguro de dotación de personal que en otras unidades de enfermería ${ }^{19-22}$. Esto puede estar relacionado con el mayor nivel de ansiedad encontrado en las enfermeras no especialistas frente a las especialistas, donde a nivel profesional pueden ser susceptibles de mayores riesgo en materia de seguridad clínica para sí, como para la seguridad del paciente siendo necesario indagar más ante los escasos estudios en este ámbito, basando los futuros trabajos en el clima de seguridad, cultura de seguridad, satisfacción laboral y percepción de riesgo de estos colectivos.

Es importante destacar que investigadores como Frutos Martin et $\mathrm{al}^{27}$ ya asocian de forma directa la ansiedad con el estrés en el trabajo y de cómo este estrés puede derivar en el síndrome de estar quemado o burnout. Atendiendo a esta idea, parece imprescindible que se implementen sistemas para monitorizar los posibles niveles de ansiedad que puedan tener las enfermeras y, desde los equipos de dirección de centros sanitarios, desarrollar acciones tales como: i) formación dirigidas a aumentar las competencias y adaptación a puestos laborales o, ii) desarrollar acciones que generen ambientes de trabajo positivos que reduzcan los niveles de estrés en el trabajo, colaborando así en el control de la ansiedad ${ }^{28}$.
Se deben mencionar las limitaciones del estudio, por un lado el procedimiento descrito se ha diseñado con el objeto de eliminar en la medida de lo posible la aparición de cualquier error no atribuible al azar o sesgo. Aunque es necesario indicar que, a pesar, de estos esfuerzos, puedan aparecer sesgos en el proceso en el que se solicita a las propias direcciones de enfermería de los hospitales que hagan llegar la información a los profesionales que ejercen sus funciones en cada uno de los hospitales; así como que los participantes que han realizado los cuestionarios de forma voluntaria tengan más afinidad que el resto de profesionales por el tema de estudio y este hecho pueda afectar a los resultados. Otra limitación del estudio es sin lugar a dudas el tamaño muestral es pequeño y solo referido a los Hospitales con Maternidad del Servicio Madrileño de Salud. Además, a la luz de los resultados obtenidos, debe destacarse la influencia del sexo en el presente estudio, donde el número de mujeres participantes fue mayor que el hombres, lo cual podría atribuirse a que la profesión enfermera, tanto a nivel de enfermeras especialistas como no especialistas, está conformada por un total de 259.129 mujeres y 48.633 hombres lo que representa un $84,19 \%$ del total del número de enfermeras colegiadas en España en el año 2018 ${ }^{30}$. A raíz de este hecho, se considera de gran interés ampliar el estudio a todo el territorio nacional con un tamaño muestral más representativo y ver si coinciden los resultados obtenidos en este estudio nacional con los que se refleja en este artículo.

Como fortalezas se destaca la novedad del estudio planteado ya que no se han encontrado estudios específicos que analicen la ansiedad en enfermeras especialistas matronas comparada con enfermeras no especialistas.

Como conclusión se puede decir que la AE es similar en ambos grupos, sin embargo, la AR en las matronas es menor que en las enfermeras no especialistas, estando ambas por debajo de la media de la población española. Este dato quizás pueda ser atribuido a la mayor formación y seguridad que las especialidades aportan, ya que las especialidades de enfermería cuentan con dos años de formación oficial específica que complementan la formación de grado de las enfermeras no especialistas, completando el desarrollo de las funciones asistenciales en enfermería. 


\section{BIBLIOGRAFÍA}

1. Real Decreto sobre especialidades de enfermería. R. D. 450/2005 (22 Abr 2005). 2. Real Decreto por el que se regula la obtención del título de enfermero especialista. R. D. 992/1987 (3 Jul 1987).

3. Real Decreto por la que se organiza la carrera de practicantes y matronas. R. D. de 10 de agosto (12 Ago 1904).

4. Martínez Martín E, Macarro Ruiz D, Herrero Martín J, Llorente Prieto A. Visión de la matrona en el Siglo XXI. Matrona Hoy. 2014; 2:17-26.

5. Willutzki U, Theismann T, Schulte D. Psychotherapy for social anxiety disorder: long-term effectiveness of resource-oriented cognitive-behavioral therapy and cognitive therapy in social anxiety disorder. J Clin Psychol. 2012; 68:581-91. 6. European Agency for Safety and Health at Work. Expert forecast on emerging psychosocial risks related to occupational safety and health. Luxembourg: Office for Official Publications of the European Communities; 2007.

7. Honkonen T, Ahola K, Pertovaara M, Isometä E, Kalimo R, Nykyri E, et al. The association between burnout and physical illness in the general populationresults from the Finnish Health 2000 Study. J Psychosom Res. 2006; 61:59-66. 8. Aiken LH, Clarke SP, Sloane DM. Nurse's reports of hospital quality of care and working conditions in 5 countries. Health Affairs. 2001; 20:43-54.

9. Costa Schmidt DR, Paladini M, Biato C, Domíngues Pais J, Rodrígues Oliveira A. Qualidade de vida no trabalho e burnout emtrabalhadores de enfermagem de Unidade de Terapia Intensiva. Rev Bras Enferm. 2013; 66:13-7.

10. Kikuchi Y, Nakaya M, Ikeda M, Narita K, Takeda M, Nishi M. Effort-reward imbalance and depressive state in nurses. Occup Med (Lond). 2010; 60:231-3.

11. Flores-Villavicencio ME, Troyo-Sanromán R, Valle Barbosa MA, Vega-Lopez MG. Ansiedad y estrés en la práctica del personal de enfermería en un Hospital de tercer nivel en Guadalajara. Revista electrónica de Psicología Iztacala. 2010; 13: 1-17. 12. Ribeiro VF, Ferreira FC, Valentí VE, Ferreira M, de Abreu LC, de Carvalho TD, et al. Prevalence of burnout syndrome in clinical nurses at a hospital of excellence. Int Arch Med. 2014; 7:22.

13. Spielberger CD. Anxiety: Currents Trends in Theoryand Research. Vol. 71 New York: Academic Press; 1972.

14. Spielberger CD, Gorsuch RL, Lushene RE. Manual for the State-Trait Anxiety Inventory. Palo Alto, CA: Consulting Psychologist Press; 1970.

15. García Batista Z, Cano Vindel A. Estandarización y validación del Inventario de Situaciones y Respuestas de Ansiedad (ISRA) en la población de República Dominicana. Summa psicológica UST. 2014; 11: 81-99.

16. Guillén Riquelme A, Buela Casal G. Actualización psicométrica y funcionamiento diferencial del ítem en el State Trait Anxiety Inventory (STAI). Psicothema. 2011; 23: 510-5.

17. Guillén Riquelme A, Buela Casal G. Versión breve del STAI en adolescentes y universitarios españoles. TerPsicol. 2013; 31:293-9.

18. Ayuso Murillo D, Colomer Sánchez A, Herrera Peco I. Ansiedad rasgo y ansiedad estado en enfermeras gestoras y clínicas de la Comunidad de Madrid. Metas Enferm. 2016; 19:21-6.

19. Orgambídez-Ramos A, Borrego-Alés Y, Ruiz-Frutos C. Empowerment, vulnerabilidad al estrés y burnout en enfermeros portugueses. Ciência \& Saúde Coletiva. 2018; 23:259-66.

20. Castillo Martínez A. Percepción de competencias en enfermeros de "roting". Index Enferm 2011; 20:1-8.

21. Cowan DT, Norman IJ, Coopamah VP. Competence in nursing practice: a controversial concept: a focussed review of literature. Nurse Educ Today, 2005; 25:355-62.

22. Dirección General de Recursos Humanos. Plan de Ordenación de Recursos Humanos del Servicio Madrileño de Salud. Consejería de Sanidad: Madrid; 2012. 23. Seisdedos N. Adaptación española del STAI. Cuestionario de Ansiedad Estado Rasgo. Madrid: TEA Ediciones; 1982.

24. Spielberger CD, Gorsuch RL, Lushene RE. STAI. Cuestionario de Ansiedad Estado-Rasgo. Madrid: TEA Ediciones; 2011.

25. Templeton GF. A Two-Step Approach for Transforming Continuous Variables to Normal: Implications and Recommendations for IS Research. CAIS. 2011; 28:4. 26. Baumann A. Entornos de prácticas favorables. Lugares de trabajo de calidad igual atención de calidad al paciente. Conjunto de instrumentos para información y actuación. Consejo Internacional de Enfermeras. 2007.

27. Orden por la que se aprueba y publica el programa formativo de la especialidad de Enfermería Obstétrico-ginecológica (matrona). Orden SAS/1349/2009 (6 May 2009).

28. Frutos Martín M, Cotado Luis MP, de la Torre Calle L, Cao Torija MJ, Castro Alija MJ, San José Arribas CA. Relación entre los modelos de gestión de recursos humanos y los niveles de estrés laboral y burnout en los profesionales de Enfermería de atención especializada. Rev Tesela. 2015;17.
29. Ayuso Murillo D, Colomer Sánchez A, Herrera-Peco I, López Peláez A. Herramientas prácticas del gestor enfermero para combatir el burnout. Rev Tesela. $2015 ; 18$.

30. Instituto Nacional de Estadística (INE) [página principal en internet]. Profesionales sanitarios colegiados 2018 [Consultado el 28 de junio de 2019]. Disponible en: https://www.ine.es/jaxi/Datos.htm?path=/t15/p416/a2018/10/\& file=s08002.px. 